\title{
LA PRESA ROMANA DE TORRETEJADA (BELALCÁZAR, CÓRDOBA)*
}

Rosa Ma. ROMERO CORRAL

\section{Resumen}

Con este trabajo, queremos dar a conocer una presa romana situada en Torretejada (Belalcázar, Córdoba).

Analizamos brevemente las presas de este tipo conocidas en la Península para centrarnos posteriormente en la de Torretejada como un ejemplo más de la infraestructura hidráulica que acompañó a la colonización agraria del ámbito rural peninsular. Ofrecemos, así mismo, nuevos datos acerca del poblamiento romano en el tradicionalmente olvidado sector Norte de la provincia de Córdoba.

\section{Summary}

With this paper we want to present a new rural Roman dam located at Torretejada (Belalcázar, Córdoba).

We analyze briefly the Roman dams of this type known in the Iberian Peninsula and after we focus on the Torretejada dam as an example of the hydraulic infrastructure which go with the Peninsular Roman rural settlement. We offer too new data about the Roman settlement in the north of the province of Córdoba, a traditionally neglected zone in studies of this kind.

Los trabajos sobre Ingeniería Hidráulica Romana han prestado poca atención al estudio de las presas; en general se han centrado en las grandes obras para abastecimiento urbano lo que ha dado lugar a una impresión errónea pues se ha pensado que

* Queremos mostrar nuestro agradecimiento a D. Francisco Godoy por indicarnos la existencia de la presa que aquí presentamos, a D. Enrique Cano profesor de la E.U.I.T. Minera de Bélmez por la realización de la topografía y a D. José Ramón Carrillo por el apoyo y la ayuda prestada para la realización de este artículo. Este trabajo se engloba en un estudio más amplio sobre el poblamiento en el norte de la provincia de Córdoba para nuestra Memoria de Licenciatura. 
son bastante escasas, interpretándose este hecho como una falta de pericia en los ingenieros romanos para construirlas. Por el contrario, los estudios más recientes revelan que se construyeron presas en todo el Imperio Romano principalmente a partir del siglo II d.C. y con mayor incidencia en ciertas áreas como el Norte de Africa, Levante y la Península Ibérica (HILL, 1984, 53); el uso y difusión de este tipo de construcciones provocó un cambio radical en cuanto al concepto y las formas de abastecimiento de agua al almacenarla directamente en la fuente (TREVOR HODGE, 1992, 87).

Tres de las más importantes obras de Ingeniería Hidráulica Romana se sitúan en España, de ellas las presas de Cornalvo y Proserpina que abastecían a la ciudad de Mérida pueden considerarse paradigmáticas y por ello tanto en sus aspectos técnicos y constructivos como en su valoración para el conjunto de la ciudad han sido tratados en numerosos trabajos (ALVAREZ 1977; SCHNITTER, 1979; FERNANDEZ, 1983, 127-137; TREVOR HODGE, 1992, 97-90); otro tanto podríamos decir de la presa de Alcantarilla (SANCHEZ, 1977) en la provincia de Toledo. También fueron utilizadas para abastecimiento urbano las presas de Consuegra (GARCÍA DIEGO-DIAZ MARTASMITH, 1980) en Toledo; la presa de Iturranduz (MEZQUIRIZ, 1984, 194-199; DIAZ et alii, 1985) y la presa de Lisboa (ALMEIDA,1969; IDEM, 1970), aunque son consideradas de menor importancia en cuanto a su calidad técnica y monumentalidad.

Fuera de la Península hay que destacar una serie de presas en el Norte de Africa, Asia Menor y Siria. Las más conocidas, debido en gran parte a la mejor conservación de los restos por el proceso de desertización que ha sufrido la zona, son las del Norte de Africa (Libia y Túnez), donde los romanos utilizaron el mismo sistema de presas para abastecimiento urbano y regadío que los nabateos habían empleado en el desierto del Neguev, zona en la que el clima y la topografía eran bastante similares; el más importante grupo de presas de esta zona se sitúa en los alrededores de Leptis Magna (VITA FINZI, 1961; SCHNITTER, 1979, 24). En Italia sólo se conocen tres que se sitúan cerca de la ciudad de Subiaco y que fueron construidas por Nerón en el río Anio para crear lagos artificiales en su villa; hay que destacar que son el único ejemplo existente de presas que no tenían un carácter utilitario; en época de Trajano fueron reutilizadas para el Aqua Annio Novus (HILL, 1984, 53).

Sin embargo, la gran mayoría de las presas romanas cortan pequeños cursos de agua de régimen irregular y secos la mayor parte del año (ALFENIM, 1992,96), y, por lo tanto, son de pequeño tamaño y de carácter rural lo que podría explicar la escasa atención que han despertado en los investigadores que se han ocupado del tema. Con la extensión del sistema de villas la red hidráulica se extendió al ámbito rural, en éste la irrigación de los campos fue una actividad bastante corriente por lo que las presas para riegos y como abrevadero para el ganado con carácter particular se convirtieron en un elemento de cierta importancia en las zonas áridas. No son muchas las publicaciones existentes para este tipo de construcciones en la Península Ibérica, aparte de algunos trabajos mas minuciosos como el de la presa de Sarral en Tarragona 
(HEREDIA-SARDA-MERCADE, 1989), el embalse de Araya en Mérida (ALVAREZ, 1970) o la presa de Aquae Flaviae (ALFENIM, 1992), la mayoría de los trabajos se limitan a mencionar su existencia en las proximidades de una villa (ALVAREZ, 1976; VIANA, 1947).

En los últimos años, debido al creciente interés por los estudios de articulación del territorio romano y sus formas de explotación se ha producido un aumento en los estudios de Ingeniería Hidráulica de uso industrial y agrícola en el ámbito peninsular. Dentro de éstos los más extensos (en cuanto al estudio de presas de uso rural) han sido los realizados en la provincia de Toledo en los que se plantea la existencia de una colonización sistemática que transformó el campo con la construcción de varias presas para uso rural (CABALLERO-SANCHEZ PALENCIA, 1982, 411; OREJAS-SANCHEZ PALENCIA, 1989, 45). Una situación semejante puede observarse para el sur de Portugal donde se han realizado varios trabajos que analizan las presas antiguas, destacando el realizado sobre las presas localizadas al sur del Tajo (CARVALHOCARDOSO-MASCARENHAS, 1989).

La mayoría de las presas romanas conocidas en la península ${ }^{1}$ se sitúan en Aragón, la provincia de Toledo, el sur de Portugal y los alrededores de Mérida. En lo que se refiere a la zona andaluza, parece que en la Bética no se hicieron muchas, al contrario de lo que se podría pensar debido a la irregularidad pluviométrica que la caracteriza. En la provincia de Córdoba podemos mencionar como excepción el Acueducto de Valdepuentes que abastecía a la ciudad de Córdoba captando el agua mediante una pequeña presa en talud en el Arroyo Bejarano (VENTURA, 1993, 61-62), si bien dista mucho de las grandes obras de Ingeniería Hidráulica como las de Cornalvo y Proserpina de Merida. Sin embargo, la mayoría de las obras hidráulicas para el abastecimiento de las ciudades en nuestra provincia captaban el agua directamente de las fuentes, como el Acueducto de Ucubi (LACORT, 1989, 376) o el de Mellaria (VAQUERIZO et alii, 1994, 156), sin recurrir a la realización de grandes obras hidráulicas. Tampoco para el ámbito rural la construcción de presas fue lo más corriente, ya que en la Campiña de Córdoba el almacenamiento de agua se realizaba mediante cisternas (LACORT, 1989; MORENA-SERRANO, 1991) exceptuando la posible presa de Almodóvar del Río² (BERNIER, 1962; PONSICH 1979, 179-180); esto podría deberse a las escasas posibilidades que ofrece el medio físico de la zona sur de la provincia para construir este tipo de obras, al carecer de una base litológica rocosa que permita su estabilidad, o bien porque la composición geológica del terreno permite el almacenamiento de agua de forma natural como es el caso de la Subbética.

\footnotetext{
${ }^{1}$ Un catálogo detallado aunque ya algo desfasado, de las presas romanas y su funcionalidad existentes en la Península Ibérica puede encontrarse en CABALLERO-SANCHEZ PALENCIA, 1982, 416-422

${ }^{2}$ En nuestra opinión no se trataría estrictamente de una presa sino de una esclusa o un muro para evitar desbordamientos. Ponsich no especifica que se trate de una presa, aunque Bernier si la considera como tal; Caballero y Sanchez Palencia la incluyen como presa en su catálogo.
} 


\section{LOCALIZACIÓN Y MARCO GEOGRÁFICO}

La presa de Torretejada tuvo un uso agrícola, como veremos mas adelante, por este motivo nos ha parecido conveniente exponer una valoración previa de las potencialidades económicas que ofrece el medio físico en la zona donde se sitúa, de este modo podremos obtener datos acerca de la forma de hábitat y la economía de la zona.

La presa se ubica en la comarca de Los Pedroches, dentro del término municipal de Belalcázar - hoja n 833 de Hinojosa del Duque - a 1²6'32" de longitud y 38³4'4" de latitud, en la curva de nivel de $500 \mathrm{~m}$ (Fig.1). Situada a $1 \mathrm{~km}$. al Norte de Casas de Torretejada, capta el agua procedente del Arroyo de Torretejada, tributario del río Guadamatilla a su vez afluente del Zújar.

Desde el punto de vista geológico se sitúa en el batolito de Los Pedroches. La base litológica sobre la que se asienta el dique está constituido por materiales Carboníferos en facies Culm compuestos de pizarras negras y gris oscuro con finas intercalaciones de grauvacas de aproximadamente $1 \mathrm{~cm}$ de ancho. Se encuentra proximo a la zona de contacto entre las pizarras y el gran cuerpo de rocas ígneas que conforman el batolito (Fig.2), compuesto por granodiorita con enclaves (RODRIGUEZ-MIRA, 1990) siendo de destacar que el vaso del embalse (alveus) se situaría sobre el granito, cuya composición facilita la retención del agua. Esta ubicación permite que la presa ċapte el agua en la zona inmediatamente anterior al punto de filtración generalizada, las pizarras, mucho mas permeables que el granito.

Como ya hemos indicado, el vaso del embalse se situaba sobre los materiales graníticos; sobre esta base se desarrollan suelos muy arenosos, con escaso contenido en materia orgánica y ligeramente ácidos (Tierras pardas meridionales sobre granitos). En el área de Sierra Morena son suelos poco evolucionados, sin embargo en la zona de Los Pedroches se ha producido una mayor descomposición del granito lo que ha permitido un mayor desarrollo edáfico, que los caracteriza como suelos ligeros y fáciles de poner en cultivo con técnicas simples (CEBAC, 1971, 186-195). Por lo tanto, dentro de la escasa calidad agrícola que tienen los suelos del norte de la provincia, la presa se sitúa sobre los que ofrecen mayor rentabilidad.

La presa se localiza, por tanto, en una zona de transición edafológica y geológica, en el área donde se produce un cambio en la base litológica que facilita la retención del agua antes de la zona de filtración. Esta situación revela unas características comunes de localización con las pequeñas presas de carácter rural de la provincia de Toledo (OREJAS-SANCHEZ PALENCIA, 1989, 50-54), por lo que repite un modelo ya documentado para la ingeniería hidráulica de época romana.

Las características agrobiológicas de este tipo de suelo están, así mismo, condicionadas por un clima mediterráneo seco en cuanto al régimen de humedad, por lo que la potencialidad agroclimática de la zona, medida en función del Indice de L. Turc, proporciona unos valores muy bajos para secano, comprendidos entre 5 y 20 , aunque aumentan considerablemente en regadío, alcanzando unos valores medios, que están comprendidos entre 45 y 55 . 
El terreno del término municipal de Belalcázar ha sido utilizado tradicionalmente para el cultivo de los cereales, siendo éste el predominante, en menor medida tambien se ha dedicado a legumbres como nos corroboran las fuentes del siglo XIX (RAMIREZ Y DE LAS CASAS DEZA, 1986). En la actualidad, la zona donde se sitúa la presa es la única donde se realizan cultivos de regadío, principalmente hortalizas, dedicándose el resto para el cereal.

\section{DESCRIPCIÓN DE LA PRESA}

La pantalla de la presa es plana (Fig.3), con una longitud de $38 \mathrm{~m}$ y una altura que varía desde $40 \mathrm{~cm}$ en los extremos hasta $2,50 \mathrm{~m}$ en su parte central (Lám.1). El dique se levanta directamente sobre los afloramientos de pizarras que le sirven de apoyo y en sus extremos está delimitado por dos suaves lomas que cerrarían el embalse, en el centro se sitúa la vaguada por donde discurre el Arroyo de Torretejada, cuyo curso es estrecho y escarpado (Fig.4).

En los extremos del dique y dispuestos casi simétricamente se sitúan dos aliviaderos de 1,10 y 1,20 m (Lám. 2) de ancho que servirían para dar salida al exceso de agua; la colocación de los aliviaderos próximos a las lomas era una nota bastante común en las presas antiguas (GARCIA DIEGO-DIAZ MARTA-SMITH, 1980, 495). Desde el centro del dique hasta el inicio de uno de los aliviaderos se conservan los restos de un revestimiento que disminuye en grosor hacia el extremo ya que la parte central es la zona de mayor altura y la que recibe mayor presión, exceptuando la parte inferior del extremo occidental en el que se puede observar un ligero aumento del espesor por razones que no hemos podido determinar; aunque no se conserve a lo largo de todo el dique pensamos que este revestimiento se extendería por casi toda la longitud de la cara de aguas abajo para ejercer mayor resistencia y contrarrestar la presión del agua embalsada, pertenecería por tanto al tipo de presas de gravedad ${ }^{3}$ (TREVOR HODGE, 1992, 80). En la actualidad el vaso del embalse se encuentra colmatado y puesto en cultivo, no obstante, hemos podido comprobar que la anchura máxima del muro es de $3,70 \mathrm{~m}$, sin tener en cuenta el revestimiento; debido a la escasa longitud y altura de la presa, lo más probable es que la cara de aguas arriba fuera recta, puesto que éste sería suficiente para soportar el empuje del agua.

El dique está construido en opus caementicium en el que se pueden diferenciar dos tongadas; los caementa son de pizarra de diversos tamaños procedentes de las cercanías del pantano, donde este tipo de roca es la predominante, como ya hemos visto más arriba; en la cara exterior del revestimiento los caementa son más regulares y de mayor tamaño que en el resto del muro.

\footnotetext{
${ }^{3}$ No se trataría de un ejemplo típico de presa de gravedad, puesto que estas suelen tener un terraplén de tierra en la cara de aguas abajo o contrafuertes para soportar la presión en la parte central; en este caso el revestimiento haría la misma función que el terraplén.
} 


\section{INTERPRETACIÓN}

Junto al vaso del embalse y en la ladera de una suave loma se han documentado varios muros (Lám. 3) y numerosos restos en superficie que nos permiten aseverar la existencia de una villa; la cronología provisional obtenida a través de la cerámica abarca desde la segunda mitad del siglo I d.C. hasta el siglo III d.C.

Las condiciones topográficas de la villa coinciden con las prescripciones que señalan los agrónomos latinos en la ubicación de las mismas (vid. FERNANDEZ, 1982, 40-51), a media altura por razones de salubridad (Catón, I,1,3; Varrón, I,12,1), en una zona relativamente fértil (Columela, I,4,5), como hemos indicado mas arriba, y provista de agua corriente (Catón, I,1,3) proveniente en este caso del arroyo de Torretejada que además se embalsaba mediante la presa para labores agrícolas de regadío.

Dado el carácter autárquico que caracterizaba las villas romanas las actividades agrarias no se concentraban en el monocultivo, pudiéndose pensar que el terreno sería explotado para cereales complementados con otro tipo de cultivos en regadío como las hortalizas o los frutales, para el autoabastecimiento o para su comercialización.

Junto a la villa se localizan los restos de, al menos, dos cisternas (Lám. 4) de opus caementicium con revestimiento de opus signinum con molduras hidráulicas que podrían surtirse del agua procedente del embalse, de manera similar a lo que se documenta en la villa de "El Hinojal" en Mérida (ALVAREZ, 1976, 445).

Las ciudades, como centros económicamente mas dinámicos son otro de los factores a tener en cuenta para la ubicación de las villas (Columela, I,2,1). En Los Pedroches se situaron dos de las pocas ciudades romanas que hubo en Sierra Morena, Solia y Baedro, pero su localización concreta sobre todo para la segunda plantea muchos problemas. Probablemente Solia se situaba en el cerro de Majadaiglesia, en el término municipal de El Guijo (STYLOW, 1985, 663) extendiéndose el territorium por la zona occidental de Los Pedroches. En el caso de Baedro, los datos son más imprecisos, aunque parece claro que se debe localizar en la zona oriental de Los Pedroches, al sur del río Guadalmez y delimitada por una serie de sierras que se extienden por la margen derecha del río (CORTIJO, 1993, 81); las inscripciones en que se menciona a baedronenses se han localizado en los actuales términos de Belalcázar, El Viso e Hinojosa del Duque (STYLOW, 1985, 660-661) por lo que la ciudad se situaría entre El Viso, Alcaracejos y la vía Corduba-Emerita, al oeste de los actuales términos de Hinojosa y Belalcázar. La villa de Torretejada se situaba por tanto dentro del radio de acción de la ciudad de Baedro.

Las principales vías de comunicación de la zona norte de Córdoba bordean por sus flancos Los Pedroches, por la zona oriental la vía Corduba-Sisapo y por la occidental Corduba-Emerita (MELCHOR, 1993, 65-70). La villa de Torretejada se encontraría por tanto alejada de las principales vías de comunicación, pero teniendo en cuenta que la proximidad de las villas a las vías de comunicación era un requisito imprescindible en el establecimiento de éstas (Catón, I,1,2) planteamos la existencia de una po- 
sible vía de carácter secundario, siendo ésta más aconsejable que las vías principales (FERNANDEZ, 1982, 49); el camino se situaría $2 \mathrm{~km}$. al sur de Torretejada y en su trazado uniría de este a oeste las dos vías principales antes mencionadas, al sur de ésta y a una distancia similar a la que tiene Torretejada con respecto al camino se ha documentado la existencia de al menos dos asentamientos de la misma época.

La escasez de lluvias propia de un clima mediterráneo seco como el que caracteriza a la zona en que nos situamos dió lugar a la creación de una infraestructura hidráulica que resolviera las necesidades de abastecimiento humano y agrario, por ello se construyeron embalses y cisternas en los que se almacenaba el agua para regar las tierras en el estío y abrevar el ganado. En general se ha asemejado la situación existente en la Península Ibérica con la conocida en las zonas áridas del norte de Africa, en donde las presas para regadío fueron una condición indispensable para la existencia de la agricultura (BELTRAN, 1977, 94), sin embargo, en opinión de algunos autores el clima de la península no convierte en indispensable el uso del regadío para el desarrollo de la agricultura (LACORT, 1989, 394). Tanto en el Norte de Africa como en la Península Ibérica el regadío no era imprescindible pero sí de gran importancia para obtener el máximo rendimiento, crear suelos cultivables y diversificar el cultivo en una zona con grandes dificultades para la agricultura (VITA FINZI, 1961, 20).

En trabajos anteriores (CABALLERO-SANCHEZ PALENCIA, 1982, 409) se aludía a la existencia de algunas zonas en Hispania que sufrieron una profunda transformación en el ámbito rural mediante obras hidráulicas, resultado de una colonización agraria sistemática. Las zonas donde mejor se conoce este fenómeno se sitúan en los alrededores de Mérida (ALVAREZ, 1976, 445; FERNANDEZ, 1983, 141) con varias presas asociadas a villas dentro del área de acción de esta ciudad; la misma situación se da en la provincia de Toledo (CABALLERO-SANCHEZ PALENCIA, 1982, 409; OREJAS-SANCHEZ,1989, 58-59). Los trabajos realizados en la Campiña de Córdoba han revelado así mismo, la existencia de una infraestructura hidráulica para la explotación agrícola del territorio, sin embargo en este caso se hizo mediante cisternas para almacenar agua (LACORT, 1989; MORENA-SERRANO, 1991). La presa de Torretejada documenta como también en el Norte de la provincia de Córdoba la implantación rural romana se vió acompañada de la construcción de obras hidráulicas que mejoraron y completaron la explotación agraria de la zona. 


\section{Bibliografía}

ALFENIM, R.A.E. (1992): “A barragem de Aquae Flaviae”. Conimbriga, XXXI, pp. 85-98.

ALMEIDA, F. (1969): "Sobre a barragem romana de Olisipo e seu aqueduto". $O$ arqueologo portuges 3, pp. 179-189.

- (1970): "Nota sobre a barragem romana de Lisboa". XI Congreso Nacional de Arqueologia, pp.693-695.

ALVAREZ MARTINEZ, J. (1970): "El embalse romano de Araya, en Merida". XI Congreso Nacional de Arqueología, pp. 729-732. Zaragoza.

- (1976): "La villa romana de El Hinojal en la Dehesa de las Tiendas (Merida)". N. A. H. 4, pp. 435-463.

— (1977): "En torno al acueducto de los Milagros de Mérida". Symposium Segovia y la arqueología romana, pp. 49-53. Barcelona.

BELTRAN MARTINEZ, A. (1977): "Las obras hidráulicas de Los Bañales (Uncastillo, Zaragoza)". Symposium Segovia y la arqueología romana, pp: 91-98. Barcelona.

BELTRAN, J.; SARDÁ, M. y MERCADÉ, L. (1989): “La presa de Sarral (Tarragona): primeros resultados del estudio arqueológico, histórico y sedimentelógico". El agua en zonas áridas: arqueología e historia. Tomo I, pp. 311-324.

BERNIER LUQUE, J. (1962): "Un fundo romano en Cuevas Bajas, Almodóvar)". B. R. A. C., pp.155-156.

BONNIN, J. (1984): L'eau dans l'antiquité. L'hydraulique avant notre ère. Paris.

CABALLERO, L. y SANCHEZ PALENCIA, F.J. (1982): "Presas romanas y datos sobre poblamiento romano y medieval en la provincia de Toledo". N. A. H. 14, pp. 381433.

CARVALHO, A.; CARDOSO, J.L. y MASCARENHAS, J.M. (1989): "Barragems antigas em Portugal a sul do Tejo". Encuentros sobre el Tajo: el agua y los asentamientos humanos, pp. 79-108. Lisboa.

CEBAC (1971): Estudio agrobiológico de la provincia de Córdoba. Sevilla.

COLMENERO, R. (1991): "O pantano romano da Abobeleira". Larouco 1, pp. 169-171.

CORTIJO CEREZO, M.L. (1993): La administración territorial de la Betica romana. Córdoba. 
DIAZ-MARTA, M.; MEZQUIRIZ, M.A.; GARCIA, N. y GARCIA-DIEGO, J. (1984): "Comentarios al estudio conjunto sobre la presa romana de Consuegra". Revista de Obras Públicas, pp.191-205.

FERNANDEZ CASADO, C. (1983): Ingeniería hidráulica romana. Madrid.

FERNANDEZ CASTRO, M.C. (1982): Villas romanas en España. Madrid.

GARCIA-DIEGO, J.; DIAZ-MARTA, M. Y SMITH, N.A.F. (1980): "Nuevo estudio sobre la presa romana de Consuegra". Revista de Obras Públicas, pp. 487-505.

GORGES, J. G. (1986): “Prospections archeologiques autour d'Emerita Augusta. Soixante-dix sites ruraux en quete de signification". R. E. A., T. LXXXVIII, pp. 215236.

HILL, D. (1984): A history of engineering in clasical and medieval times. London.

LACORT NAVARRO, P. (1989): "Obras hidráulicas e implantación rural romana en la Campiña de Córdoba". El agua en zonas áridas: arqueología e historia. Tomo I, pp. 361-401. Almería.

MELCHOR GIL, E. (1993): "Vías romanas y explotación de los recursos mineros de la zona Norte del conventus cordubensis". A. A. C. 4, pp. 63-89.

MEZQUIRIZ IRUJO, M.A. (1985): "Nuevos datos sobre la presa romana de Iturranduz". M. Z. B. 4, pp. 163-166.

MINISTERIO DE AGRICULTURA (1977): Mapa de cultivos y aprovechamientos. E./1:50.000. Hinojosa del Duque.

MORENA, J.A. y SERRANO J. (1991): "Obras hidráulicas romanas en la campiña oriental de Córdoba (Baena-Cañete)". II Encuentros de Historia Local. La Campiña I, pp. 117-138.

OREJAS, A. y SANCHEZ-PALENCIA, F.J. (1989): "Obras hidráulicas romanas y explotación del territorio en la provincia de Toledo". El agua en zonas áridas: Arqueología e Historia. Tomo I, pp. 45-63. Almeria.

PONSICH, M. (1979): Implantation rurale antique sur le Bas-Guadalquivir. Paris.

RAMIREZ Y LAS CASAS DEZA, L.M. (1986): Corografia histórico-estadística de la provincia y obispado de Córdoba (ed. de A. López Ontiveros). Tomo I. (or. de 1867).

RODRIGUEZ PEVIDA, L. y MIRA LÓPEZ, M. (1990): Mapa geológico de España. E. 1/50.000. Hinojosa del Duque.

SANCHEZ ABAL, J.L. (1977): "Obra hidráulica romana en la provincia de Toledo (Pantano de Alcantarilla)”. Symposium Segovia y la arqueología romana, pp. 359-366. Barcelona. 
SCHNITTER REINHARDT, N. (1979): "Les barrages romains". Doss. Arch. 38, pp. 20-25.

STYLOW, A.U. (1985): "Ordenación territorial romana en el Valle de Los Pedroches (Conventus Cordubensis)". XVII Congreso Nacional de Arqueología, pp. 657666. Zaragoza.

TREVOR HODGE, A. (1992): Roman aqueducts and water supply. London.

VAQUERIZO, D. et alii (1994): El Valle Alto del Guadiato (Fuenteobejuna, Córdoba). Córdoba.

VENTURA VILLANUEVA, A. (1993): El abastecimiento de agua a la Córdoba Romana. El acueducto de Valdepuentes. Córdoba.

VIANA, A. (1947): "Hidraulica agricola na epoca romana". Arquivo de Beja 4, pp.18-23.

VITA FINZI, C. (1961): "Roman Dams in Tripolitania". Antiquity XXXV, pp. 1420. 


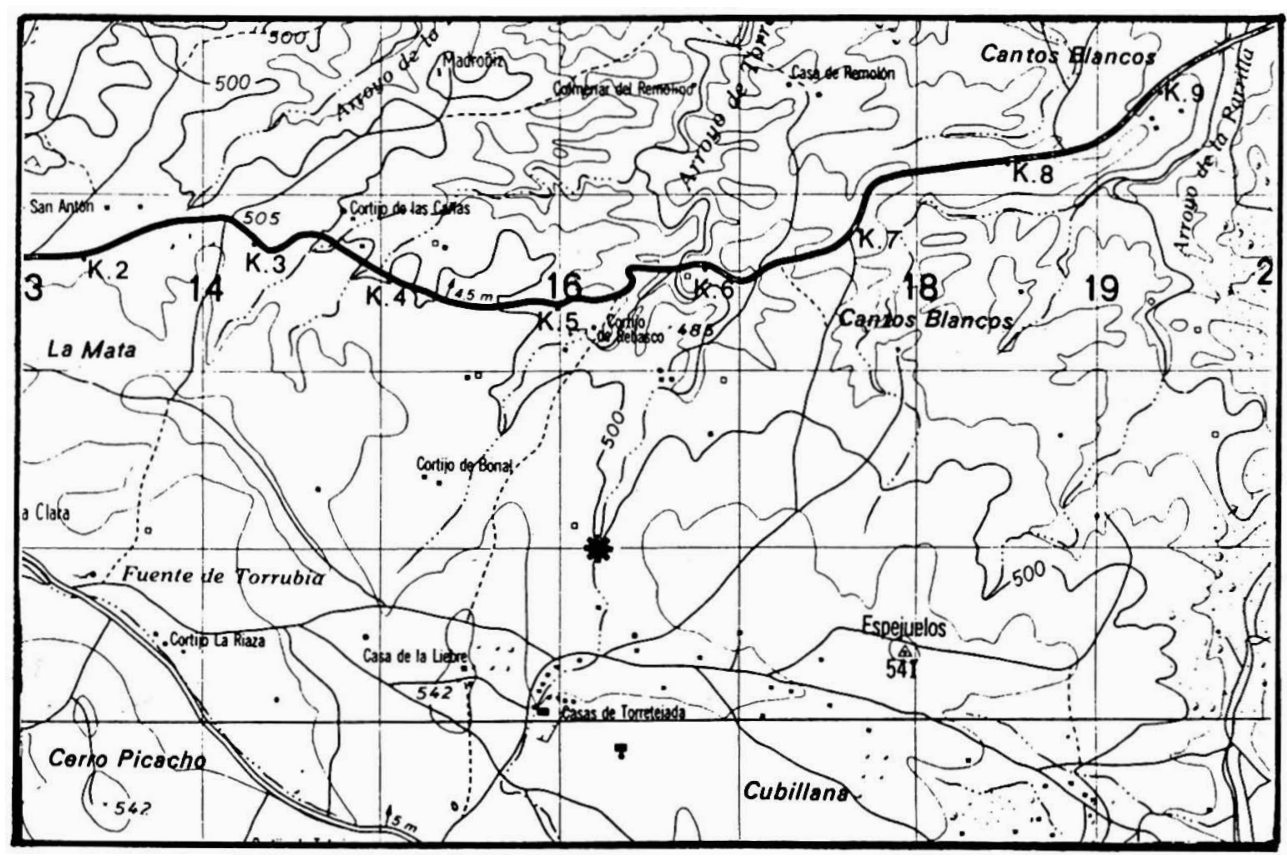

Fig. 1. Localización de la presa de Torretejada. E 1/50000

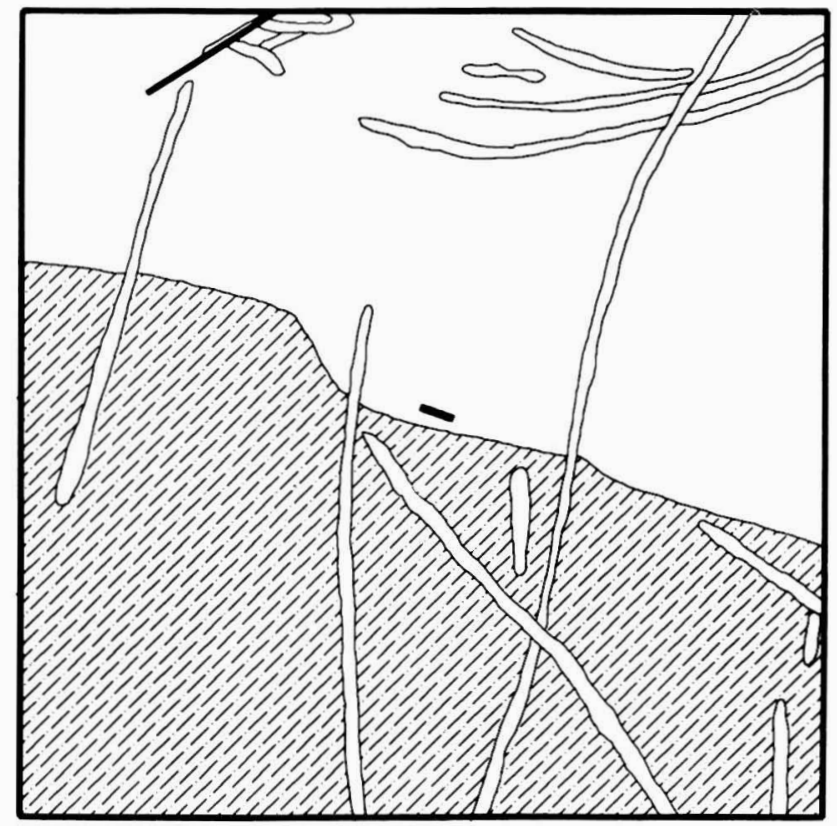

Fig. 2. Esquema geológico (la presa aparece en la zona central). E 1/50000. 

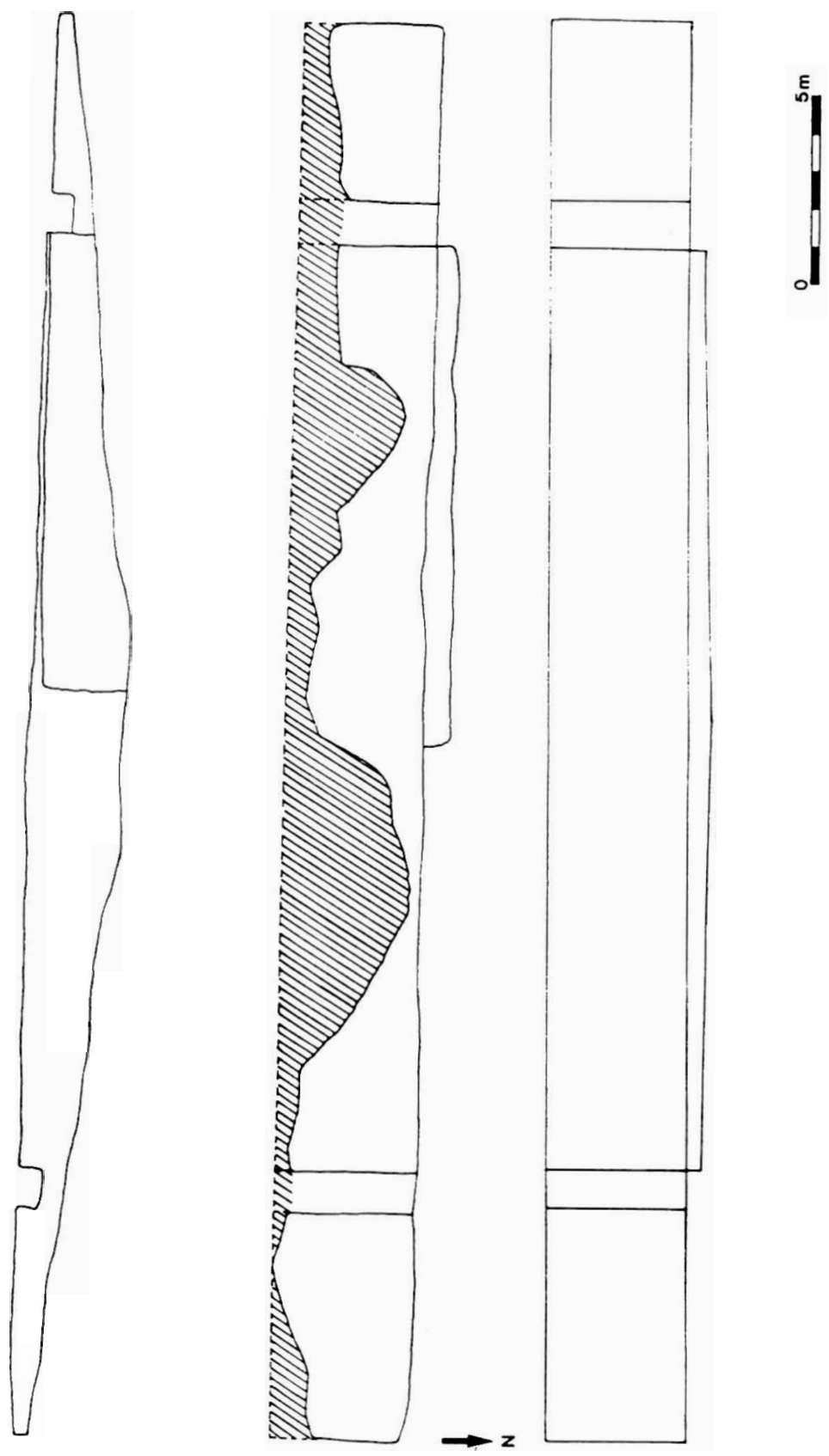

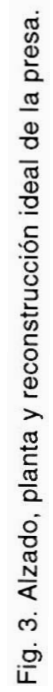




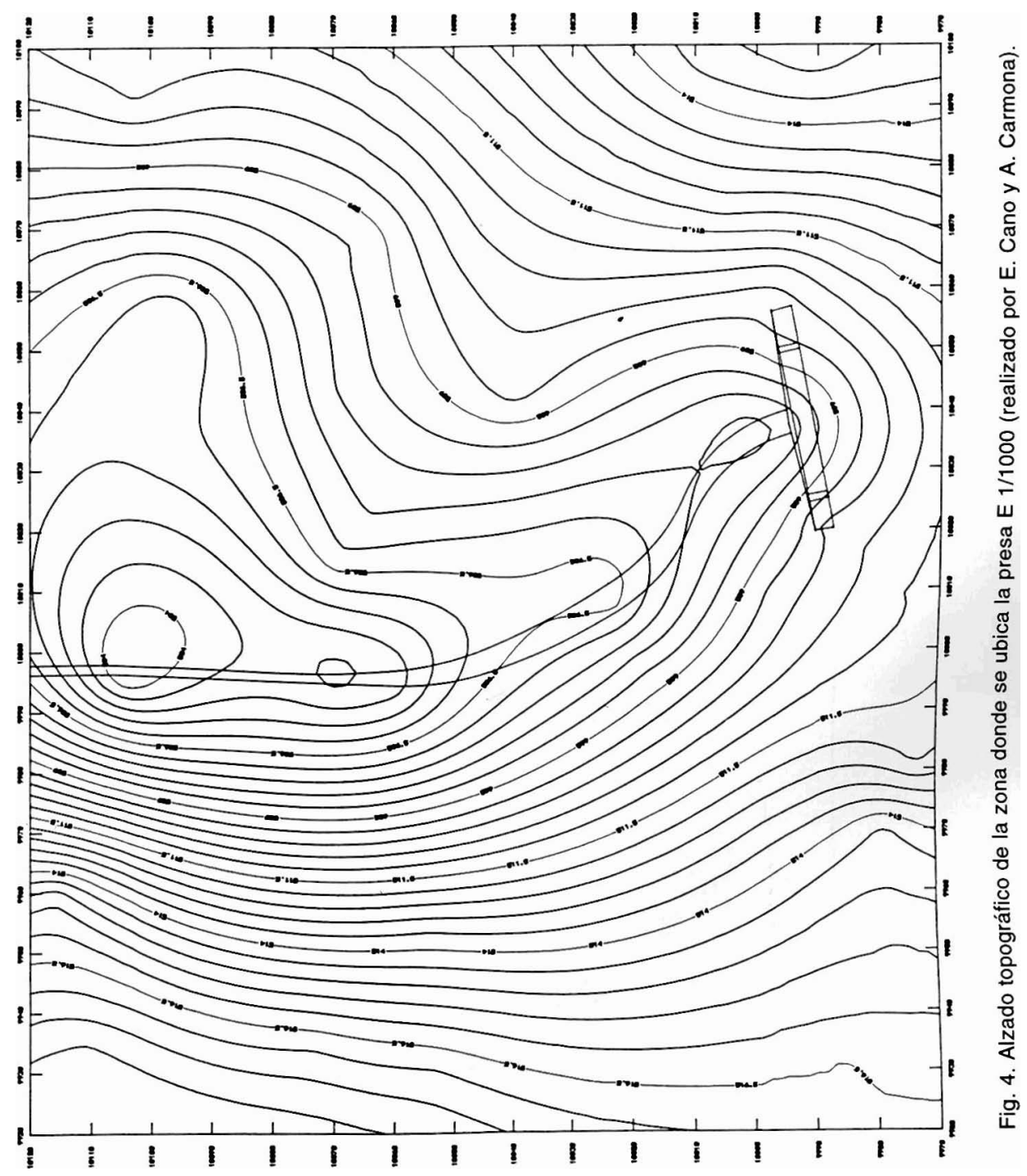




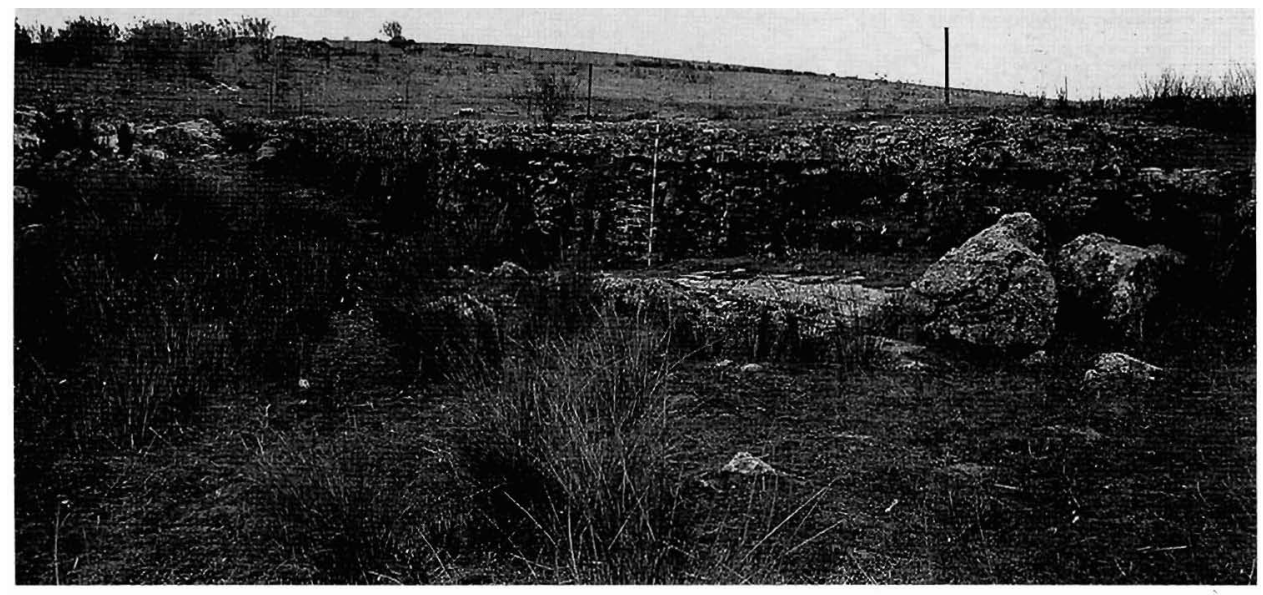

Lám. 1. Vista general de la presa desde la cara de aguas abajo.

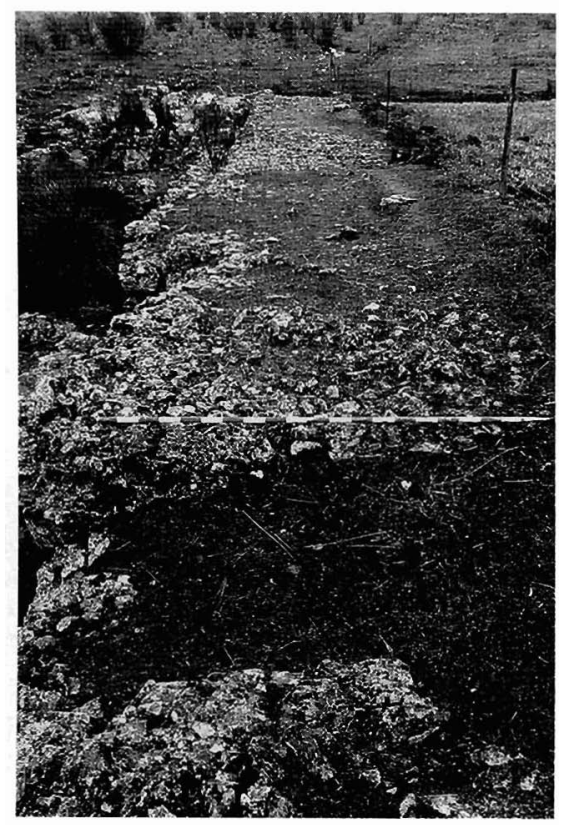

Lám. 2. Aliviadero del lado este. 


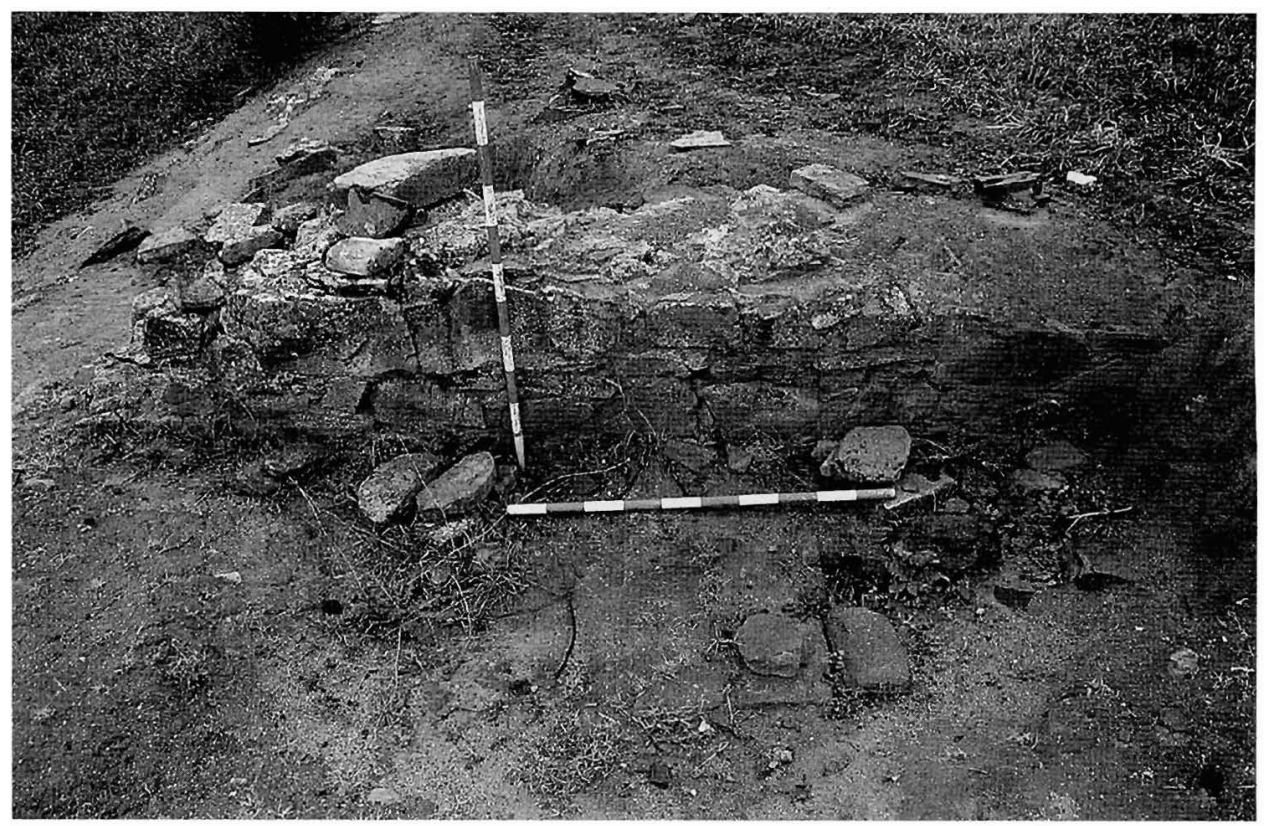

Lám. 3 Restos de muros perenecientes a la villa.

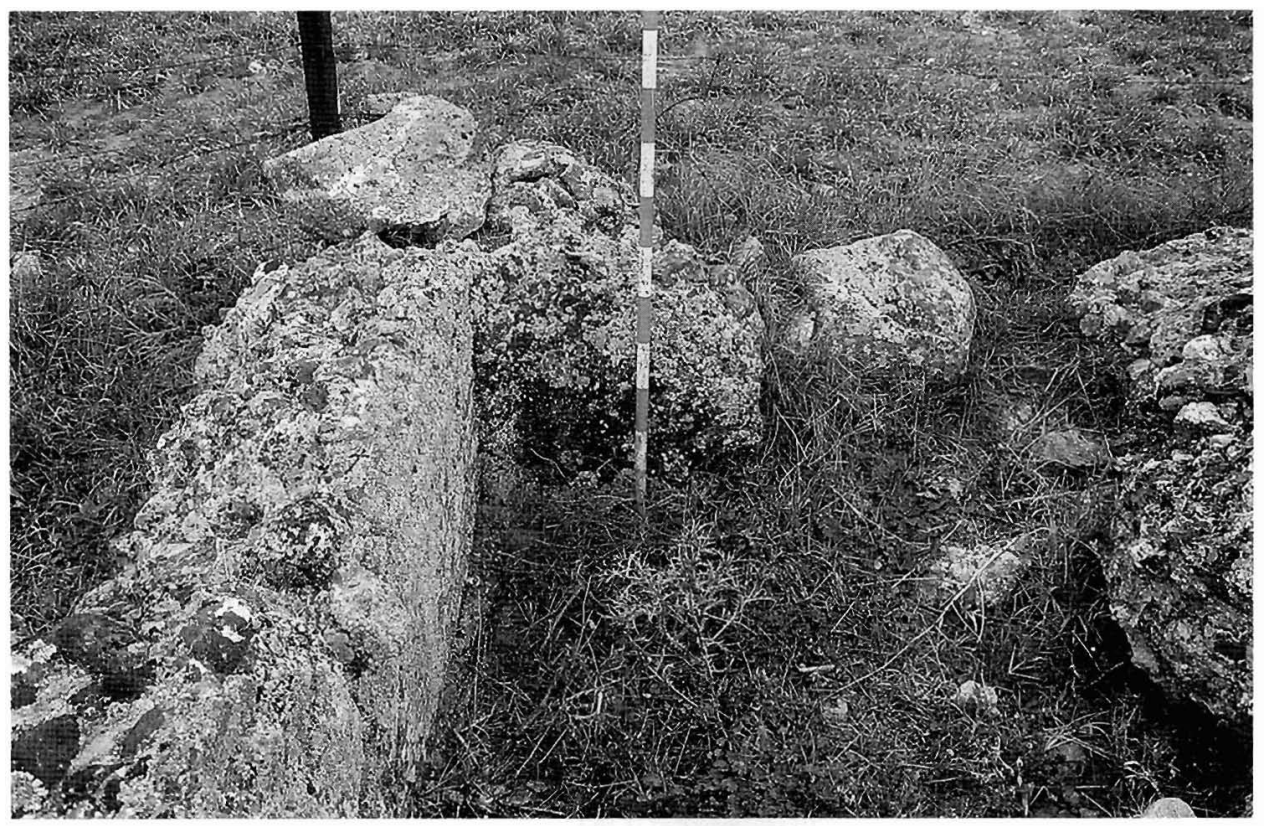

Lám. 4. Restos de las cisternas. 